Authors: Tiago Silva ${ }^{*}$, Vera Fernandes ${ }^{1,2,3^{*}}$, Diana Martins ${ }^{5}$, Diana Gonçalves ${ }^{6}$, Raquel Almeida ${ }^{7}$, Ana Margarida Monteiro ${ }^{1}$, Carolina Neves $^{8}$, Helder Simões 9,10 , Pedro Marques ${ }^{11}$, Filipa Serra ${ }^{10}$, Maria Lopes Pereira ${ }^{1}$ e Grupo de Estudos de Tumores da Supra-Renal.

Hospital: 1 Department of Endocrinology, Hospital de Braga; 2 Escola de Ciências da Saúde, Universidade do Minho; 3 ICVS/3B's - Laboratório Associado, Braga/Guimarães; 4 Department of

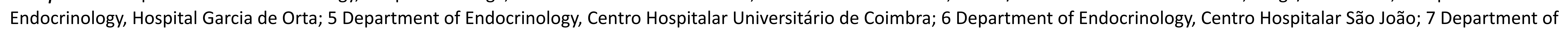

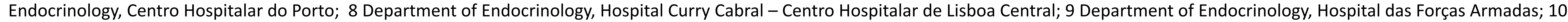
Department of Endocrinology, Hospital de Egas Moniz - Centro Hospitalar de Lisboa Ocidental; 11 Department of Endocrinology, Instituto Português de Oncologia de Lisboa

\title{
INTRODUCTION AND OBJECTIVES
}

Primary Aldosteronism (PA) is the most prevalent cause of secondary hypertension. In Portugal there is no multicentre study characterizing PA patients. The aim of this study was to characterize the diagnostic workup, treatment and follow-up of patients with confirmed PA.

\section{METHODS}

The adrenal tumour study group of the Portuguese Society of Endocrinology undertook the first retrospective multicentre study of PA patients. The inclusion criteria were a positive aldosterone/renin ratio and a positive confirmatory test. Data was gathered from 9 Portuguese Endocrinology centres. The data was analysed with SPSS $21^{\circ}$.

\section{RESULTS}

\begin{tabular}{l|c|c}
\hline Characteristics & Mean \pm SD/ & Min-Max \\
\hline Age at diagnosis (years), $\mathrm{n}=62$ & $52,1 \pm 13,1$ & $18-78$ \\
\hline $\begin{array}{l}\text { Age of hypertension diagnosis } \\
\text { (years), } \mathrm{n}=54\end{array}$ & $41,1 \pm 10,6$ & $16-68$ \\
\hline Hypertension duration $\mathbf{n = 5 5}$ & $9,9(7-16)$ & $0,6-45$ \\
\hline $\mathrm{IMC}\left(\mathrm{Kg} / \mathrm{m}^{2}\right), \mathrm{n}=35$ & $26,1(23,9-29,4)$ & $21,5-33,8$ \\
\hline
\end{tabular}

\begin{tabular}{lc}
\multicolumn{1}{c}{ Baseline laboratory values } & Mean \pm SD/ \\
Serum aldosterone $(\mathrm{ng} / \mathrm{dL}), \mathrm{n}=63$ & $33,4(20,4-53)$ \\
\hline Urine aldosterone $(\mathrm{mcg} / \mathbf{2 4 h}), \mathrm{n}=23$ & $42(25-59)$ \\
\hline ARP $(\mathrm{ng} / \mathrm{mL} / \mathrm{h}), \mathrm{n}=18$ & $0,2(0,2-0,2)$ \\
\hline Aldosterone/ARP, $\mathrm{n}=20$ & $97,1(74-336,3)$ \\
Serum K (mmol/L), $\mathrm{n}=60$ & $3,2 \pm 0,7$ \\
\hline Creatinine $(\mathrm{mg} / \mathrm{dL}), \mathrm{n}=58$ & \\
\hline $\begin{array}{l}\text { Dexamethasone supression test } 1 \mathrm{mg} \% \text { positive } \\
\text { tests, }(>1,8 \mathrm{ng} / \mathrm{mL}) \mathrm{n}=17\end{array}$ & $0,77(0,7-0,9)$ \\
\hline
\end{tabular}

Global treatment results (\%)

60

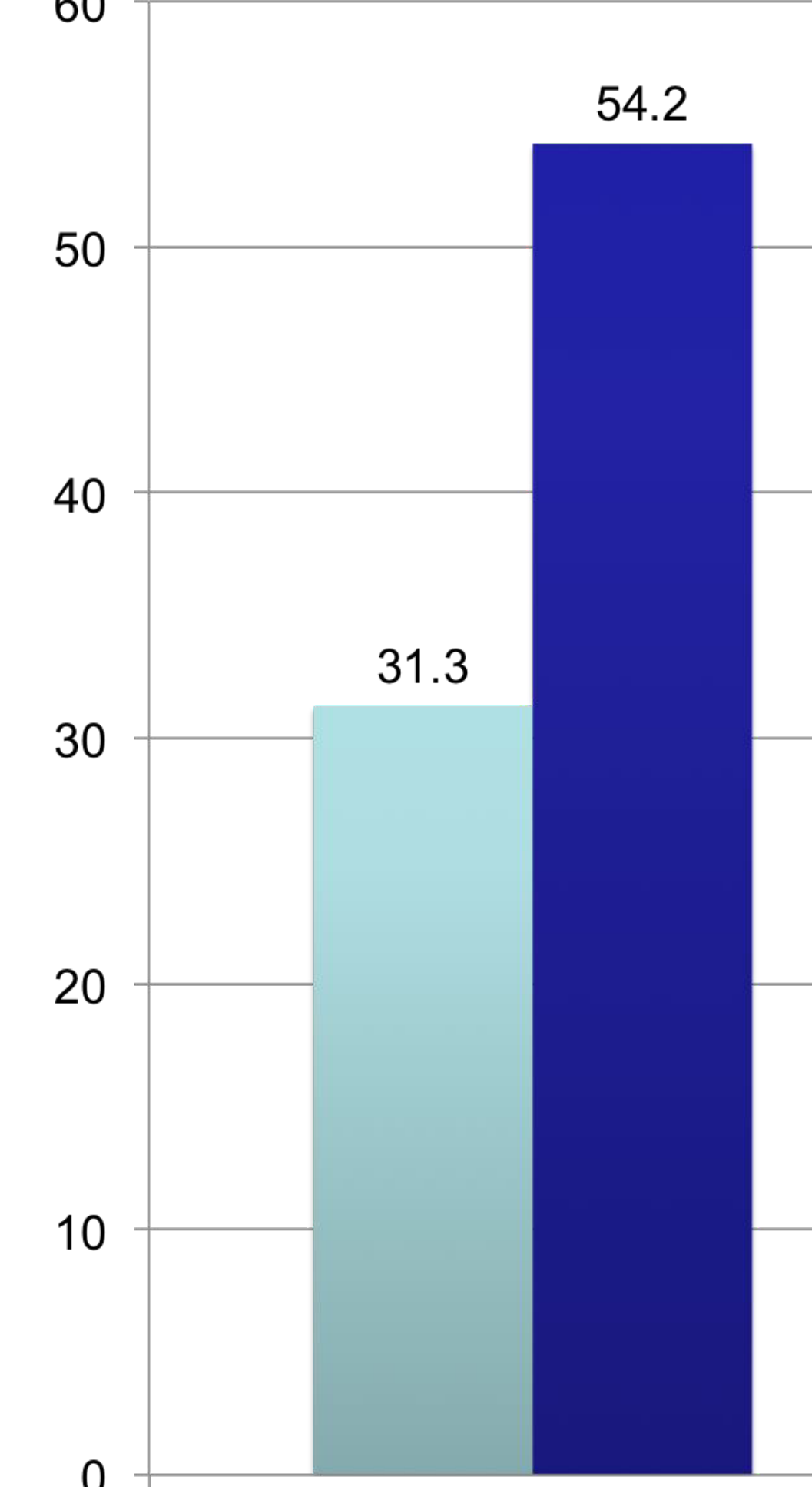

HYPERTENSION

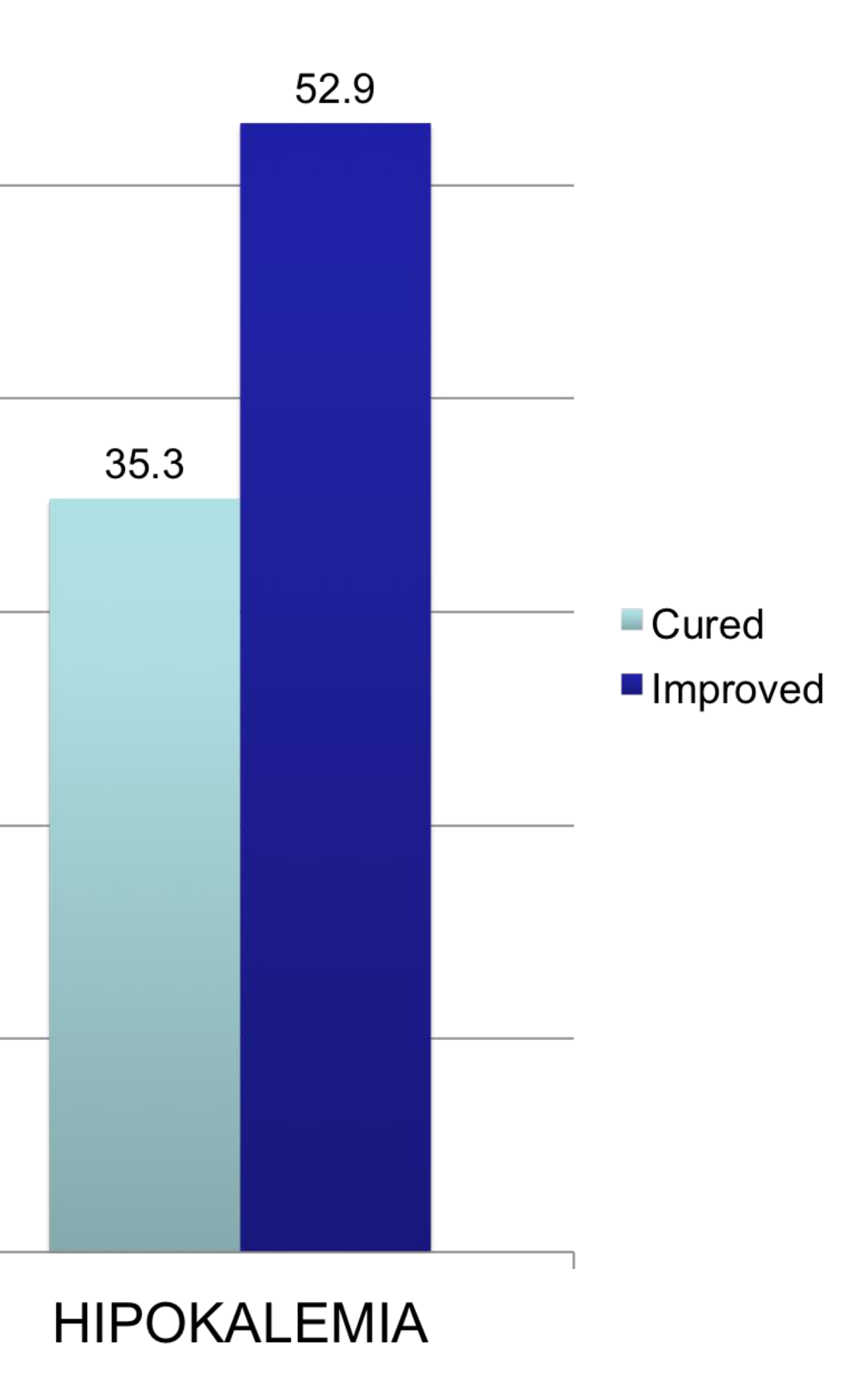

CONCLUSIONS
Form of presentation (\%)
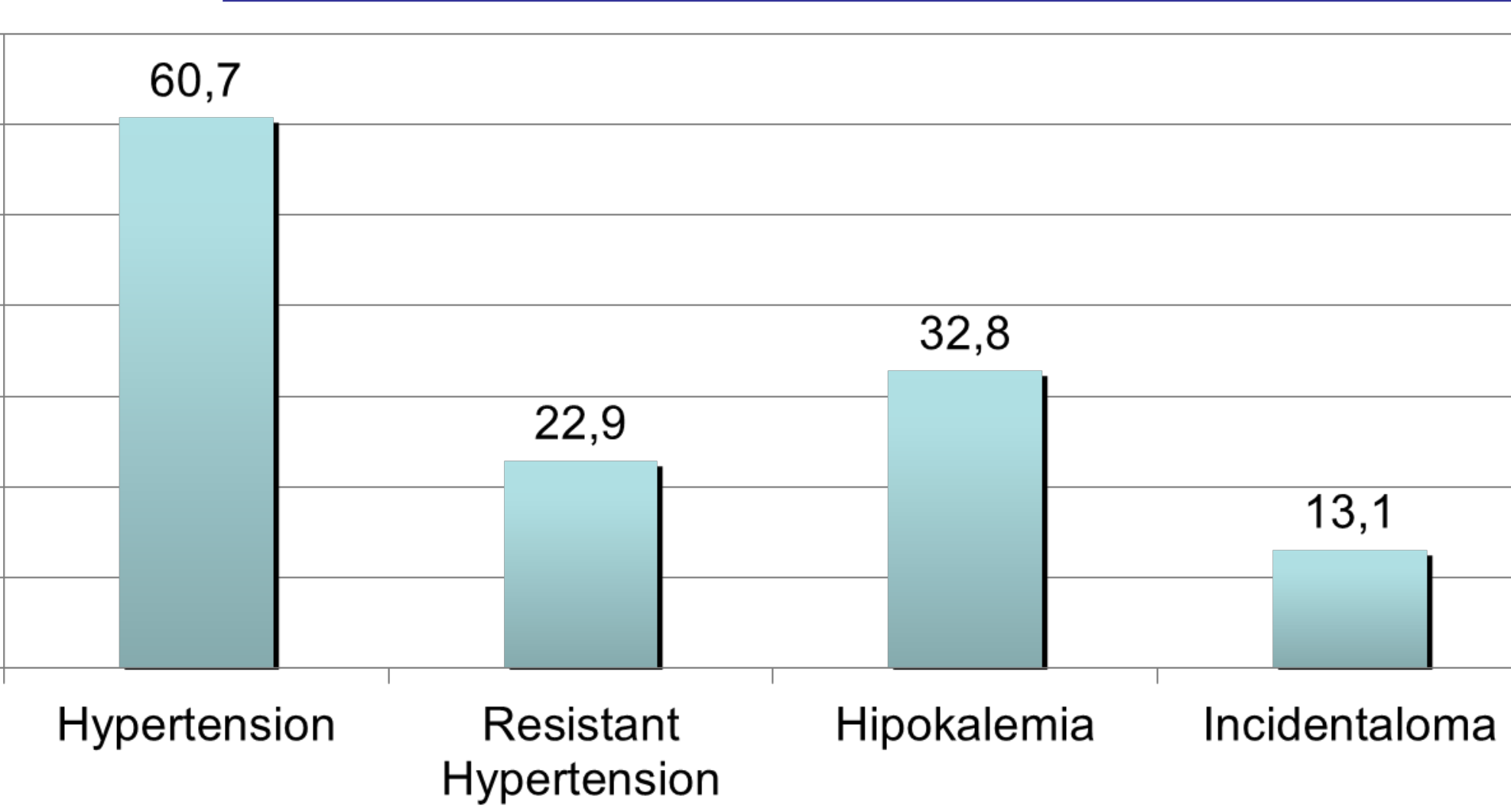

\section{Confirmatory tests (\%)}

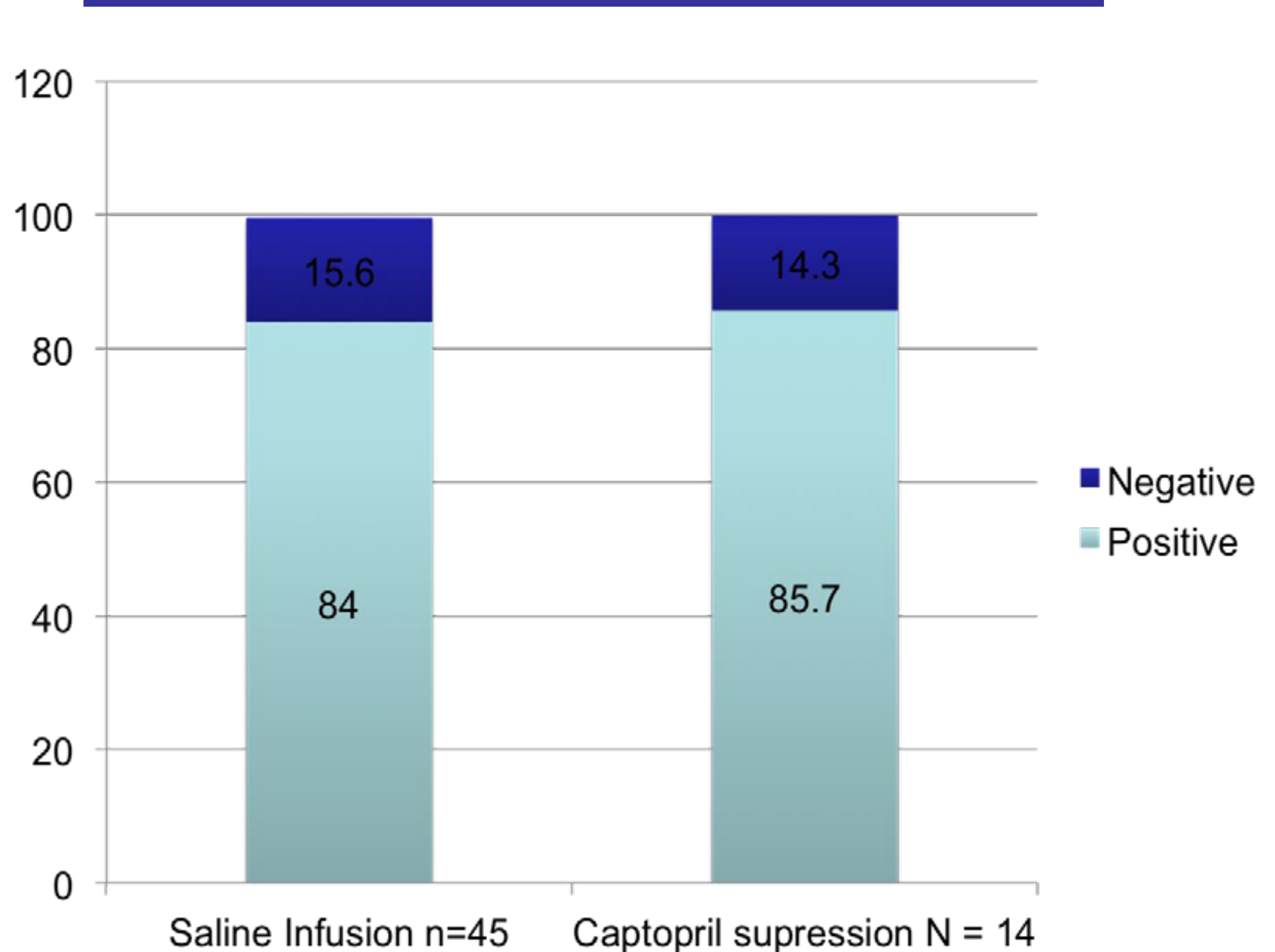

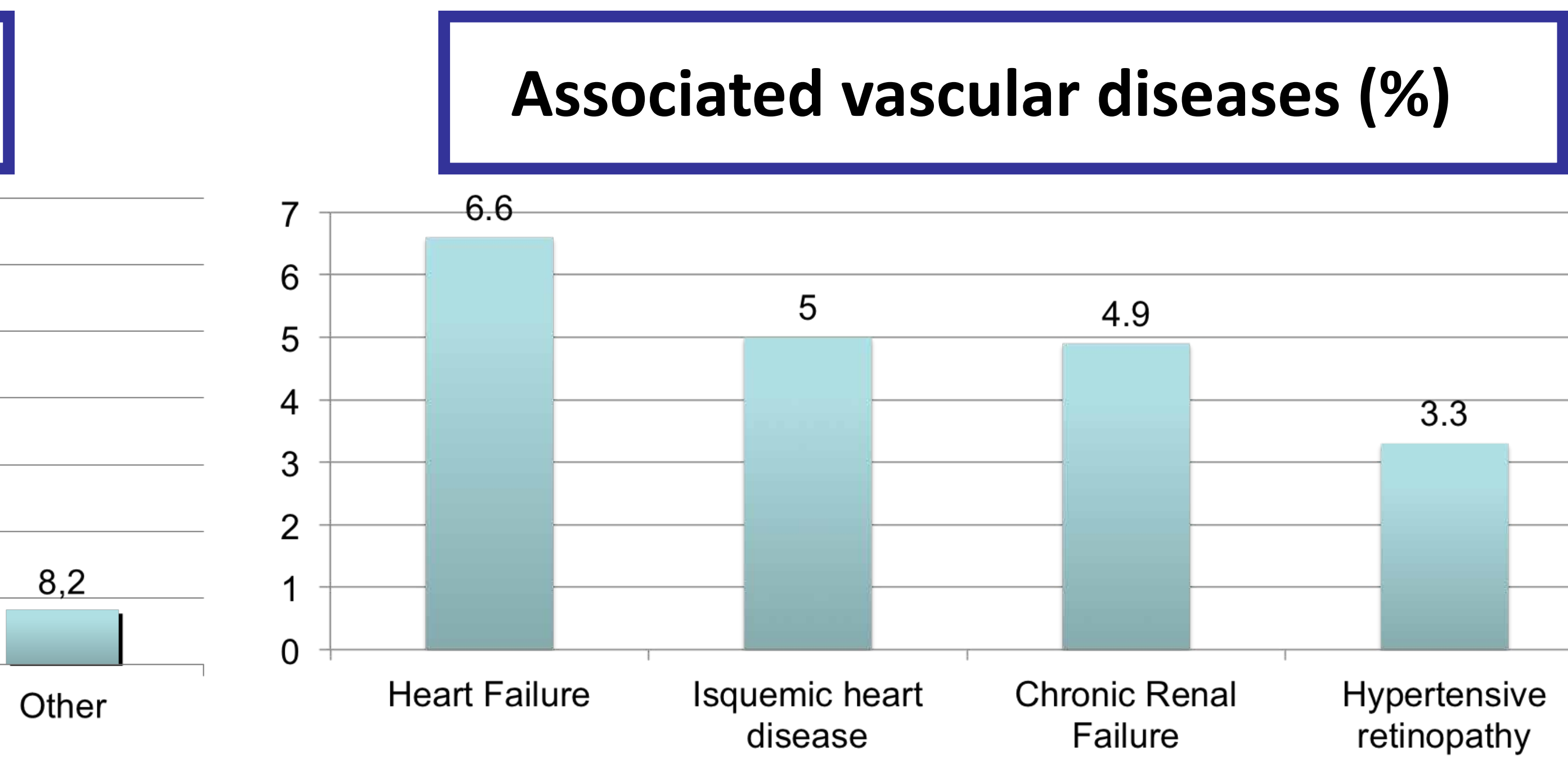

Imaging method

Results

$88,9 / 11,1$

$87,3 / 3,2$

$86,4 / 13,6$

$1,7(0,7-8,3)$

Nodule size in cm (median min-max)

Arterial venous sampling $(n) n=62$ Successful (n)

9

1

lodocholesterol scintigraphy (n) $n=62$

Unilateral fixation /no fixation (\%)

Agreament between lodocholesterol and CT (\%)

14

$64 / 29$

50

Differences between surgical and medical treated patients

\begin{tabular}{|c|c|c|c|}
\hline Variable & $\begin{array}{c}\text { Surgery } \\
(58,3 \%)\end{array}$ & $\begin{array}{c}\text { Aldosterone } \\
\text { Antagonists } \\
(\mathbf{4 1 , 7 \% )}\end{array}$ & $\mathbf{P}$ \\
\hline $\begin{array}{c}\text { Age at diagnosis } \\
\text { (years) }\end{array}$ & $47 \pm 9,3$ & $54,2 \pm 15,8$ & 0,074 \\
\hline $\begin{array}{c}\text { Sex (female) } \\
\text { Hypertension }\end{array}$ & 78,6 & 50 & 0,038 \\
\hline Treated hypertension & 100 & 42,1 & 0,002 \\
\hline
\end{tabular}

Duration of

Hypertension (years)

Tumor size $(\mathrm{cm})$
$8(4-13)$

$14(10-21,5)$

0,002

$1,8(1,5-2,4)$

$1,5(1-1,8)$

0,022

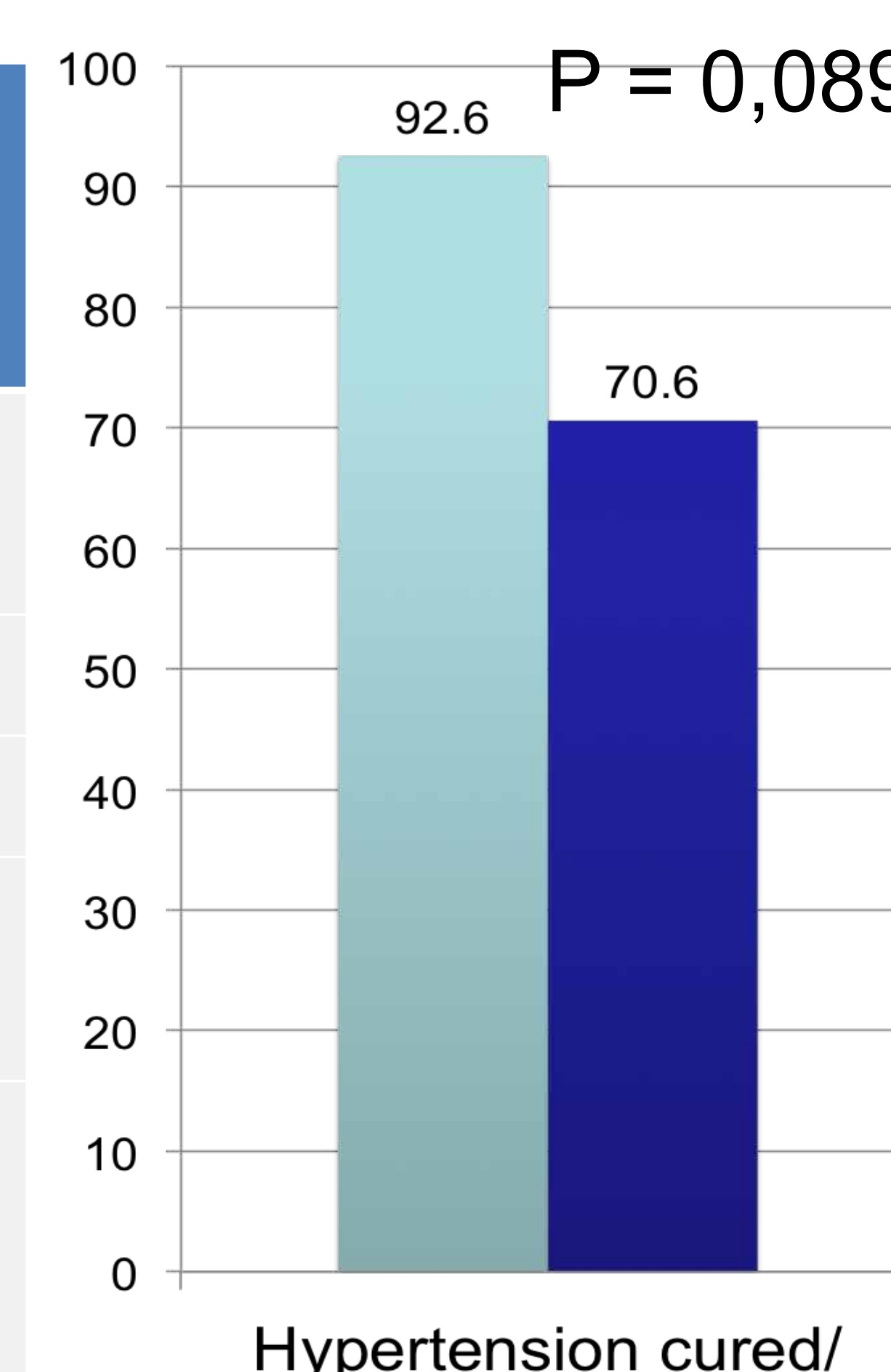

Hypertension cured improved

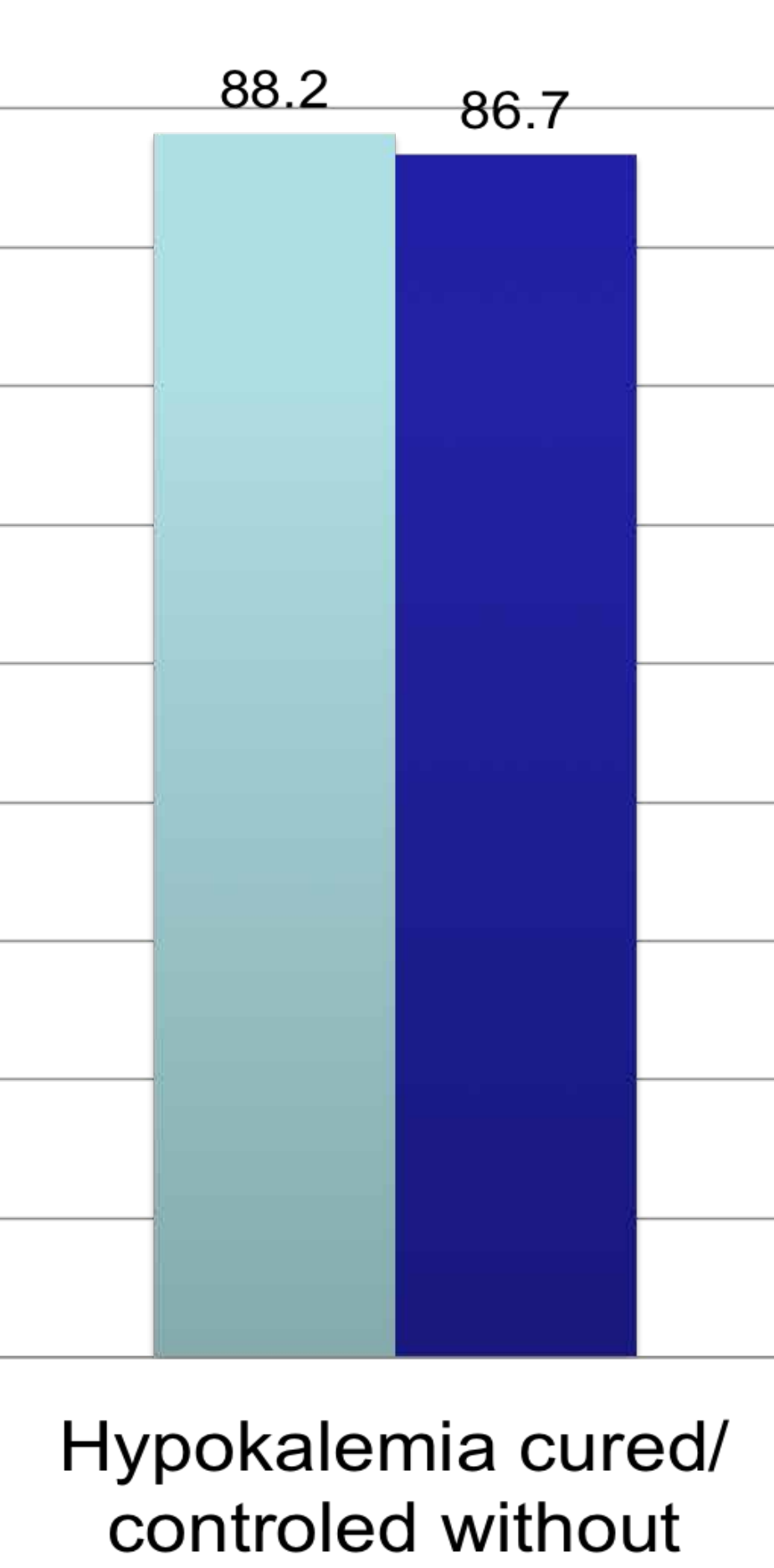

controled without
- Sugery

- Aldosterone antagonists

REFERENCES

This is the first Portuguese PA multicentre study. It shows that PA remains an underdiagnosed condition with a significant delay in diagnosis. Surgical treated patients were younger, had more hypertension, a longer duration of hypertension and bigger adenoma size with a trend towards better hypertension control.

\section{1}

,

\#Funder JW et al. Case detection, diagnosis, and treatment of patients with primary aldosteronism. I Clin Endocrinol Metab. 2008; 93(9): 3266-81.

\#Mulatero P, et al. Increased diagnosis of primary aldosteronism, including surgically correctable forms, in centers from five continents. JCEM. 2004; 89(3):1045-50.

\#Catena C et al. Cardiovascular outcomes in patients with primary aldosteronism after treatment. Arch Intern

\#Born-Fronsberg Eet al. Card primary aldosteronism: results of the German Conn's Registry. JCEM. 2009; 94(4):1125-30. 